\title{
Un vocabulario para la indización del mundo profesional del Antiguo Régimen
}

\author{
María Rosa Santos Lorite \\ Universidad de Zaragoza
}

\subsection{Resumen}

Ante la variedad de términos utilizados en fuentes documentales de la Zaragoza del Siglo XVI para designar profesiones se plantea la necesidad de la normalización de los mismos de modo que posibilite el tratamiento y recuperación posterior de la información, facilitando la realización de estudios posteriores sobre la historia de la lectura. Tras el examen de algunos tesauros y vocabularios de indización existentes, valorando la conveniencia de su aplicación, se descarta esta posibilidad y se opta por la realización de un instrumento de indización propio. (Autor)

Palabras clave: Profesiones. Indización. Tesauros.

\subsection{Abstract}

In the presence of the variety of terminology used in document sources from Zaragoza in the sixteenth century to designate professions, the need to normalize them in a way that makes possible the treatment and later recovery of the information appears. This facilitates carrying out later studies about the history of reading in the Zaragoza of the Renaissance. The possibility of application of some of the already available thesauri and indexing vocabularies has been evaluated, taking into account the suitability, and rejected. The preparation of an indexing instrument has been the chosen option. (Author)

Keywords: Professions. Indexing. Thesaurus.

\section{Introducción}

A la hora de organizar el mundo profesional del siglo XVI, tratando de sistematizar la riqueza que aparece en las fuentes documentales de la época y la variedad de oficios y de términos para hacer referencia a dicha realidad extraida de documentos notariales contemporáneos, nos interesa determinar en qué medida son válidos vocabularios o tesauros existentes y si es preciso elaborar algún instrumento de control terminológico propio. 
La utilidad de la realización de un estudio de estas características, como veremos, es la de organizar una realidad, la de las ocupaciones del siglo XVI en Zaragoza, para obtener representaciones de la misma que faciliten trabajos posteriores. Es decir, para el estudio de la lectura en la sociedad, es importante establecer la incidencia de ella en diferentes grupos sociales. Por ejemplo, a fin de constatar en qué medida aparecen los libros entre los bienes de un colectivo profesional o qué libros pueden caracterizar a dicho colectivo (bibliotecas profesionales), es necesario realizar un control y sistematización. Si tomáramos sólo los términos de los documentos tal como aparecen, se dispersaría la información.

El origen de este trabajo está en la necesidad de organizar la información obtenida a través de fuentes primarias historiográficas, documentos contemporáneos al período y asunto objetivo central de nuestra investigación. Las ciencias documentales aquí jugarán un papel decisivo en el tratamiento de una información (input) sometida a un proceso de normalización y organización que posibilite su recuperación posterior (output). En la consulta de las fuentes documentales que nos ocupan, los inventarios de bienes contenidos en los protocolos notariales de la segunda mitad del siglo XVI en Zaragoza (1) se proporcionan normalmente datos sobre los otorgantes de documentos, y en este caso de los propietarios de los bienes inventariados y de los propios bienes. En el registro correspondiente a cada inventario estableceremos dos categorías de datos de interés. El primer grupo es el relativo al propietario, el segundo a los bienes recogidos en el inventario. En este primer grupo los campos de dicho registro serán los siguientes: nombre, apellidos y alias, si lo hubiese del propietario de los bienes, domicilio, sexo y profesión. En el segundo caso se procederá a dividir los bienes en grupos según su naturaleza.

La justificación de nuestro trabajo es transformar los datos extraídos de los documentos para lograr una normalización básica que permita pasar de lo individual a lo general, y obtener conclusiones para el estudio de la lectura en Zaragoza en el siglo XVI, y en concreto, en este caso, permiténdonos ver su caracterización por sectores de la sociedad. Como pone de relieve M. J. Pedraza (1999, p.152): $\mathrm{El}$ inventario ofrece una muy importante información sobre el lector, como se ha visto. El nombre, la profesión, la parroquia o barrio donde vive —en ocasiones la calle — y su familia y allegados - ejecutores. Todos estos datos permiten agrupar a los lectores por sexos, por profesiones, y por los barrios en los que viven. De esta manera se pueden establecer aproximaciones a las características sociológicas de los lectores... Los datos obtenidos permiten una aproximación al lector desde una perspectiva general mediante la clasificación de los lectores a partir de sus características más sobresalientes.

Nos incumbe el tema de las ocupaciones puesto que es primordial para cualquier estudio posterior y sistematización de datos obtenidos. En nuestro caso interesaban las bibliotecas y la lectura, así que obviamente para llegar a conclusiones acerca de grupos sociales era preciso primero establecerlos de forma uní-

Scire. $8: 1$ (en.-jun. 2002) 131-145. 
voca. Los lenguajes documentales, sean tesauros o clasificaciones, pueden y deben auxiliar al investigador en la elaboración de sus productos.

\section{Objetivos y metodología}

El objetivo a alcanzar era, por tanto, la búsqueda de un tesauro, o bien, la creación de un vocabulario controlado de términos propio, que facilitaran la elaboración de productos posteriores, como índices o bases de datos, en el seno de la realización de trabajos de investigación a partir de la consulta de las fuentes historiográficas. Se trata de facilitar el tratamiento y recuperación de la información obtenida a partir de dichos documentos.

En consecuencia, se parte de la consulta de fuentes documentales y de los conceptos y términos obtenidos en ellas, a sistematizarlos de cara a un estudio subordinado a otros. La primera fase de este estudio pasa por la recogida de los términos tal y como figuran en la documentación, con toda su complejidad y riqueza. Se incluirán las variaciones ortográficas y las sinonímicas. Tras la consulta de bibliografía e instrumentos documentales, veremos si éstos son de aplicación, las dificultades que surgen, etc. Finalmente, elaboraremos un vocabulario de indización que pueda sernos de utilidad, y cercano en algunos aspectos a un tesauro, pero con un nivel de complejidad básico y adaptado al fin para el que lo concebimos. Se trata de la propuesta de un vocabulario de indización realizado a partir de los términos del lenguaje natural recogidos en los documentos notariales del siglo XVI en Zaragoza, siguiendo, por tanto, un método inductivo, basado en la recopilación de los términos en un momento previo a la elaboración del tesauro.

\section{La utilización de tesauros existentes}

La opción por un tipo de lenguaje documental como el tesauro, es una alternativa que valoramos antes de realizar este trabajo. Se consideró la posibilidad de utilizar algunos tesauros ya existentes determinando si se adaptaban y respondían a las necesidades planteadas en la recopilación y tratamiento de la información de los documentos consultados. El tesauro, lenguaje documental de estructura combinatoria, tiene ventajas como "su flexibilidad, capacidad de especialización, la multiplicidad de combinaciones que permite establecer entre los términos de su vocabulario, el alto nivel de control terminológico y su facilidad de revisión y puesta al día" (Gil, 1996, p. 354), ventajas que lo convierten en una herramienta idónea para los objetivos propuestos.

Un primer ejemplo que consideramos interesante examinar es el del denominado Vocabulario de indización y consulta de la base de datos BADATOR, por estar concebido para el tratamiento de registros basados en documentos de archivo y haber seguido un método principalmente inductivo para su elaboración,

Scire. 8: 1 (en.-jun. 2002) 131-145. 
aspecto en el que coincide con nuestro tema. Se ha partido también de los documentos y en el caso de BADATOR de las descripciones de los mismos realizadas en instrumentos archivísticos, y desde el lenguaje natural empleado en ellos. En este caso la diversidad terminológica procede más de la variedad de archivos y de personas que han elaborado los inventarios, en que se basan los registros de la base de datos, que de la de los documentos, como ocurre en nuestro caso, aunque esta diversidad será muchas veces producto de las fuentes documentales. La recogida de información sobre Euskadi existente en archivos ubicados fuera de esta comunidad autónoma, se inició en 1989 (2) por parte del Centro de Patrimonio Documental de Euskadi (IRARGI). El trabajo se centró en los dos Archivos con documentación cualitativamente más importante: los Procesos civiles del Archivo de la Real Chancillería de Valladolid y la Sección de Consejos Suprimidos, en el Archivo Histórico Nacional de Madrid. El proyecto recibió el nombre de Badator en 1991, y concluyó en 1997, con un resultado cercano a las 40.000 fichas de vaciado recogidas correspondientes a los siglos XIII al XIX, con esta ingente cantidad de información, y la derivada de trabajos de normalización de archivos vascos, se ha diseñado el Programa Badator, cuyo objetivo es difundir toda la información de todos los Fondos de Archivos relacionados con el País Vasco. Badator actúa como un intermediario que difunde información de numerosos fondos de archivo. El vocabulario o tesauro resultante excede por su riqueza de términos y temática lo que sería un vocabulario elaborado para controlar como materia las profesiones y ocupaciones de la sociedad del Antiguo Régimen. No obstante, un examen del mismo nos revela que aparece, como era lógico, un número considerable de descriptores relativos a ocupaciones, cargos, etc. Dichos términos están distribuidos a través de los capítulos integrantes de su listado jerárquico de materias (3). En definitiva, de las diez categorías principales o capítulos de la estructura jerárquica de BADATOR, en nueve de ellas aparecen términos referidos a oficios y cargos. No obstante, donde la densidad de éstos es mayor es en el capítulo de economía, donde se reproduce la tradicional división por sectores de la actividad económica. Recoge bastantes términos de los que aparecen en nuestra documentación, pero a veces se han seleccionado como formas aceptadas algunas más actuales frente a las más frecuentes en la documentación del siglo XVI, es el caso de veterinario, que se hace prevalecer frente a albéitar, término más cercano al de los documentos.

Es un trabajo interesante, como hemos dicho más arriba, porque trata de responder a las necesidades de un sistema de información concreto, $\mathrm{y}$, desde ese punto de vista es un modelo de método de trabajo similar al que se debe seguir para obtener un listado controlado de descriptores aplicables al mundo profesional del siglo XVI en Zaragoza. Pero las diferencias derivadas de la finalidad y origen del tesauro desaconsejan su uso para la organización de los términos relati-

Scire. 8: 1 (en.-jun. 2002) 131-145. 
vos a ocupaciones de la Zaragoza renacentista.

Además de éste, también se tomaron en consideración tesauros (4) en los que apareciesen profesiones, sea de modo central, como en el de la OIT, sea entre otros campos semánticos. Se llegó a la conclusión de que, en nuestro caso, ninguno se adaptaba plenamente a nuestras necesidades y resultaba conveniente elaborar un instrumento de indización propio.

\section{La lista de términos y su normalización}

Llegado este punto, parece lógico concluir que no existen tesauros fácilmente aplicables al objetivo propuesto y es más razonable construir nuestro propio vocabulario de indización de profesiones del siglo XVI en Zaragoza, extraído de la documentación y normalizado hasta obtener un listado de descriptores con sus términos descartados y sus relaciones. Resulta demasiado ambicioso considerarlo un tesauro, si bien en lo posible hemos respetado la normativa para la construcción de tesauros monolingües (5).

La primera tarea a realizar es la recopilación de los términos en un listado. En esta lista aparecen todos los términos referidos a ocupaciones, cargos, etc., que hemos extraído de la consulta de la fuente primaria de información, los propios documentos, los protocolos notariales y, dentro de ellos, de los inventarios de bienes, donde, como ya se ha dicho, se proporcionan normalmente datos del propietario de los mismos incluyendo su ocupación. El primer listado se elaboró sin ningún tipo de discriminación, es decir, con la inclusión de todos los términos recogidos con las correspondientes variantes ortográficas y con todos los sinónimos.

La segunda fase exigía la realización de un listado en el que hubiese un control de los términos conducente a la obtención de términos unívocos de manera que a cada término correspondiera un único significado y que cada significado fuera representado por un único término, descartando la sinonimia propia del lenguaje natural. En este momento se agrupan las variantes de expresión tanto ortográficas como sinonímicas que se han encontrado para el mismo significado y se opta por una de ellas como descriptor (6). En la lista resultante se distribuyen los términos en tres categorías: forma aceptada, variantes ortográficas de la forma aceptada y formas excluidas. La primera incluye, por lo tanto, los términos preferentes; la segunda, variantes que no pueden ser consideradas como términos no preferentes, puesto que es un problema de ortografía y no de sinonimia, son los mismo términos con distintas formas de las que una sola es elegida, lógicamente la más cercana a la grafía actual; y la tercera, muestra los términos sinónimos y cuasisinónimos del término preferente (7). Ante la disyuntiva de usar un término simple, o bien, uno compuesto, se ha seguido la recomendación de la norma UNE, epígrafe 7, optando por unitérminos siempre que ello no supusiera problemas de comprensión.

Scire. 8: 1 (en.-jun. 2002) 131-145. 
Por ejemplo, entre librero y mercader de libros, se ha elegido la primera forma.

El primer escollo con el que nos encontramos en el camino hacia la consecución de un lenguaje documental de términos unívocos, una vez finalizada la recopilación de los mismos, es la gran variedad de formas ortográficas que aparecen. Este primer problema lo salvaremos optando por la forma más cercana a la grafía actual y de mayor uso entre los historiadores. Pero incluso en este punto, aparentemente poco conflictivo, sería deseable contar con el auxilio de un lenguaje documental que sirviera de autoridad y que fuese de uso generalizado (8), de este modo resulta más fácil el intercambio de conocimientos entre diversos profesionales de una materia y es algo de lo que se adolece en las ciencias sociales. "La característica más significativa del lenguaje histórico, es sin duda alguna la imprecisión. La variedad de significados es un tema propio de la historiografía, y ante la que se encuentra el documentalista a la hora de convertir este lenguaje en otro más controlado y preciso..” (Rubio, 1999, p.23).

En el momento de abordar la documentación histórica aplicándole técnicas documentales que permitan el tratamiento de la información que esta proporciona, es preciso tomar en consideración el hecho de que presenta características propias. Como indica Rubio Liniers (Ibidem, p. 29-30), los historiadores utilizan el documento como fuente primaria de su investigación y toman como fuentes primarias los documentos coetáneos al momento histórico estudiado, especialmente el documento de archivo, como ocurre en nuestro trabajo:

Generalmente, el concepto de documento y documentación va unido para el historiador a la documentación de archivo. Este es, por supuesto, su origen etimológico y conceptual, ya que documentar y documentación han sido definidos durante siglos como dar fe de algo. Así el documento era en sí mismo la prueba de la veracidad del pasado... Se advierte cada vez más la necesidad de un análisis de contenido de los documentos como el mejor sistema de recuperación temática y conceptual. La mayoría de los archivos históricos nacionales se están planteando la elaboración de tesauros para el análisis de sus fondos.

El segundo problema es el de la sinonimia, de significativa incidencia en nuestro léxico. En principio, son frecuentes algunas de las variantes de sinonimia lingüística que enumera Chaumier (1986) como son la variante ortográfica (por ejemplo, naipero y naypero) la equivalencia entre forma antigua y moderna (por ejemplo, albéitar y veterinario) y la sinonimia semántica (médico, doctor en medicina y físico). En estos casos lo normal será optar por una de las formas (siempre teniendo en cuenta que la sinonimia completa no es frecuente) y en el índice envíar desde los términos no elegidos al preferente. En aras de la economía de términos se evitará, en lo posible, incrementar el número de ellos con cuasisinónimos de uso menor en los documentos.

No hemos encontrado apenas problemas de polisemia puesto que el ámbito de nuestro vocabulario es lo suficientemente restringido para que no aparezcan 
complicaciones de este tipo. Por ejemplo, el término corredor, sin su ubicación en este contexto, podría presentar problemas en cuanto a su significado existiendo diversas acepciones para el mismo, con su delimitación semántica, dada por su inclusión en un léxico de ocupaciones de la Zaragoza del siglo XVI, las posibilidades de interpretación se reducen y el significado queda claro, sin que sea preciso recurrir a métodos como el uso de calificadores, para deshacer la polisemia. Sólo en algunos casos puntuales incluimos aclaraciones entre paréntesis para delimitar los términos, pero dichos casos son excepcionales. Para un ejemplo de término polisémico como camarero, el método de desambiguación escogido ha sido el de añadir un determinante entre paréntesis: Camareros (de iglesia)

Los problemas de delimitación semántica afectarán, sobre todo, a la clasificación, aspecto que sería de interés en una fase de construcción del lenguaje documental en la que se abordaran relaciones jerárquicas entre términos, algo que excede los objetivos iniciales de nuestro vocabulario. Es el caso de muchos oficios gremiales en los que una misma persona, por ejemplo un sombrerero o un zapatero, realiza tareas de fabricación, de venta y comercialización de sus productos. Actualmente, las funciones suelen estar separadas y quienes comercializan no tienen que ser fabricantes, pero la realidad que intentamos clasificar se resiste a entrar en nuestros esquemas. Un zapatero sería a la vez fabricante y comerciante de zapatos y esto lo sitúa en dos lugares de la clasificación a la vez, sectores secundario y terciario, lo cual suele obviarse a efectos prácticos. Para la forma de los términos usaremos normas ISO-AENOR correspondientes, prefiriendo el uso del plural, teniendo en cuenta que los términos que denominan profesiones son sustantivos concretos y cuantificables. En la ortografía de los términos, la fuente es el Diccionario de la Lengua Española, de la Real Academia Española de la Lengua, y no se han aceptado las variaciones ortográficas por lo que no se harán reenvíos desde las mismas. Además, indicar que cuando se han incorporado términos compuestos su orden es el del lenguaje natural. En cuanto al género, optamos por el masculino genérico (9). Como resultado de este proceso y de la aplicación de los criterios y normas hasta aquí expuestos, se obtiene la lista definitiva de términos para la indización de profesiones de la Zaragoza del siglo XVI.

\section{Propuesta de un vocabulario de indización para profesiones del siglo XVI en Zaragoza}

La lista definitiva de términos para la indización de las profesiones, que servirá como herramienta de control terminológico y normalización del campo de ocupaciones de nuestra base de datos de inventarios, se ha realizado incluyendo algunas de las relaciones del tesauro, como son la de equivalencia entre términos preferentes y no preferentes, y la asociativa. Para su presentación se han utilizado las abreviaturas y formas que aparecen en la norma UNE. La presentación es

Scire. 8: 1 (en.-jun. 2002) 131-145. 
alfabética, es decir, con todos los términos de indización, preferentes o no, formando parte de una secuencia alfabética única. En ella, con cada descriptor se incluye el conjunto completo de sus relaciones semánticas.

Una característica que se puede apreciar en la lista de términos y en el vocabulario o lista de descriptores resultante, es que las distintas profesiones no tienen el mismo peso específico en el vocabulario, y en algunas se distinguen más categorías dentro, o "especializaciones" de las mismas, como en el caso de los corredores, o los tejedores. La razón de que se haya producido este resultado es fruto del método utilizado en su elaboración, a partir de los documentos. Esto explica que algunos colectivos no aparezcan, pese a tener una presencia real en la sociedad, como sería el caso de los pobres. La lista alfabética de términos de indización (10) para oficios del siglo XVI en Zaragoza se incluye en el apéndice 1 (11).

\section{Conclusiones}

Podemos afirmar, finalmente, que en este trabajo se han cumplido los objetivos que nos habíamos propuesto en un principio. Tras el examen de los tesauros especializados y otros vocabularios de indización concluimos que su uso resulta inapropiado para las necesidades expuestas y que la opción lógica es la realización de una herramienta propia. En este punto la clave del problema radica en que un tesauro se elabora para unas necesidades específicas y no siempre puede aplicarse a otras. Como exponen Aranes y Landa (2000, p. 634):

[...] habremos de ser conscientes de lo que deseamos conocer u obtener informativa y documentalmente del ámbito abordado, y por tanto habremos de explicitar lo que necesitamos controlar y para qué: de qué modo y con qué recursos (fines y medios). Según qué respondamos a estas preguntas — cargadas de raíz epistemológica—, diseñaremos un sistema u otro de tratamiento documental y, sobre todo, sabremos quiénes somos y con qué objeto y de qué manera deseamos conocer e intervenir sobre la realidad... Después de la aplicación del sistema, seremos conscientes de lo que hemos obtenido, como y en qué medida representamos y conocemos la realidad.

Un caso claro sería el del Tesauro OIT, totalmente orientado al mundo del trabajo, pero al actual, por lo que la distancia entre las realidades a representar y a las que aplicar la herramienta terminológica, determina su escasa utilidad fuera del contexto para el que ha sido concebido. Si nuestro trabajo fuese para una organización del mundo ocupacional actual, el tesauro OIT habría resultado idóneo. Por tanto, a la hora de plantearse el uso de un tesauro, es conveniente revisar las opciones existentes, por si alguna se acomoda a nuestras necesidades. Pero en el caso de que éstas se alejen de nuestros objetivos, deberemos elaborar nuestro instrumento de trabajo.

En nuestro caso, la necesidad de realizar un vocabulario de indización propio se hizo pronto patente. Pese a que se trata de un modesto instrumento pensado 
para la organización de una información y una base de datos de uso personal, pero en la que se basará una investigación conducente a la obtención de conocimiento sobre la lectura en la Zaragoza renacentista, no es un trabajo innecesario, pues puede ser de vital importancia para la obtención de los resultados fiables a partir de la documentación consultada. Además de esta utilidad, la elaboración de un instrumento de control terminológico es un excelente ejercicio de reflexión acerca de la importancia del léxico para la ciencia y en qué medida nuestro conocimiento de la realidad depende de las representaciones que elaboramos o recibimos de la misma.

Como cualquier vocabulario de indización, el nuestro está sujeto a revisión para su actualización en lo relativo a la incorporación de nuevos descriptores y no se ha descartado realizar una estructura jerárquica para el mismo, aunque eso lo determinará su utilización, que es la que da la medida de la satisfacción obtenida en su uso como herramienta.

\section{Notas}

(1) Conservados en su mayor parte en el Archivo Histórico de Protocolos Notariales de Zaragoza, donde se ha consultado en la actualidad más del $90 \%$ de los mismos.

(2) La información sobre BADATOR procede tanto de la obra coordinada por Martín Suquía (1993), como de la consulta de su sede virtual http://www.irargi.org/01default.htm

(3) Se presentan las materias en un listado jerárquico, acompañado de una representación gráfica, y otro alfabético.

(4) Los tesauros examinados son los siguientes: Tesauro de Historia Contemporánea, Tesauro ISOC de Economía, Tesauro SPINES, Tesauro UNESCO y Tesauro OIT.

(5) La norma ISO 2788-1986.

(6) O término preferente, que es el "que se utiliza sistemáticamente en la indización para representar un concepto determinado", según la norma UNE 50-106-90.

(7) Son términos no preferentes. El término no preferente se define así en la Norma UNE 50-106-90: "El sinónimo o cuasisinónimo de un término preferente. Un término no preferente no se asigna a los documentos, pero proporciona un punto de acceso a partir del cual el usuario es dirigido mediante una intrucción (por ejemplo, USE o VÉASE) al término preferente apropiado; el término no preferente se conoce también como no-descriptor."

(8) Una exposición clara del problema de la necesidad de conceptualización denunciada por los especialistas en Documentación que han investigado el lenguaje en Ciencias Sociales y Humanas y de las dificultades de control de vocabulario a las que se enfrentan, la encontramos enRubio Liniers (1999, p. 19-23).

(9) En el caso que nos ocupa, inventarios del siglo XVI, no sería razonable duplicar los accesos añadiendo términos en femenino. No por ello se debe pensar que restamos importancia a la documentación relativa a mujeres, pero la realidad de las fuentes con las que trabajamos es que no aparecen términos de ocupación en femenino. Cuando

Scire. $8: 1$ (en.-jun. 2002) 131-145. 
el inventario pertenece a una mujer los datos que acompañan a la misma suelen ser los relativos a su estado civil (viuda relicta de, doncella, mujer que fue de) y, en algunos casos, la profesión de su cónyuge. La única excepción sería la de criada o la de viuda que, raramente, aparece caracterizada con la profesión del marido en femenino. Para estos casos, en el supuesto de utilizar un tesauro se deberían contemplar remisiones desde los términos de género femenino que vayan apareciendo, incorporándolos al lenguaje documental conforme sea necesario.

(10) Las abreviaturas utilizadas son USE (que precede al término preferente) y UP (Usado Por, precede a término no preferente), para las relaciones de equivalencia y TR (Término Relacionado), para la relación asociativa.

(11) Lógicamente, no es una lista cerrada y se encuentra sujeta a modificaciones y a ingreso de nuevos términos en ella a medida que los mismos sean necesarios.

\section{Apéndice I: Vocabulario de profesiones del Antiguo Régimen}

Abades

Abejeros

UP Apicultores

UP Colmeneros

Abogados fiscales

TR Doctores en derecho

TR Juristas

Adobadores de cueros

USE Pelliceros

Agricultores

USE Labradores

Aguadores

Aguardenteros

Agujeros

Alabarderos

Aladreros

Albañiles

TR Obreros de villa

Albéitares

UP Veterinarios

Alcaides

Alguaciles

Aljeceros

Almadieros

Alquiladores de mulas

TR Correos

TR Trajineros
Andadores

Apicultores

USE Abejeros

Apotecarios

USE Boticarios

Arcabuceros

TR Armeros

TR Ballesteros

TR Escopeteros

TR Espaderos

TR Polvoristas

TR Puñaleros

Archiveros

Arciprestes

Armeros

TR Arcabuceros

TR Ballesteros

TR Escopeteros

TR Espaderos

TR Polvoristas

TR Puñaleros

Arrieros

Artífices de pintura

USE Pintores

Arzobispos

TR Obispos

Atadores de libros

USE Encuadernadores
Autores de comedias

Ayudantes de andador

Azabacheros

Azulejeros

Ballesteros

TR Arcabuceros

TR Armeros

TR Escopeteros

TR Espaderos

TR Polvoristas

TR Puñaleros

Barberos

TR Cirujanos

TR Médicos

Barqueros

UP Maestros de hacer

barcas

TR Carpinteros

Bataneros

Batidores de oro

Blanqueros

TR Pelliceros

TR Pergamineros

TR Zurradores

Boneteros

TR Sombrereros

Bordadores

Boteros

Scire. 8:1 (en.-jun. 2002) 131-145. 


\author{
Boticarios \\ UP Apotecarios \\ UP Farmacéuticos \\ TR Drogueros \\ Buidadores \\ TR Estañeros \\ Caballerizos \\ Cabreros \\ TR Ganaderos \\ TR Pastores \\ TR Vaqueros

\section{Cajeros} \\ Calceteros \\ TR Satres \\ Caldereros \\ Camareros(de iglesia) \\ Campaneros \\ Canónigos \\ Cantareros \\ Canteros
}

UP Maestros de cantería

Cañameneros

UP Cañamicero

TR Esparteñeros

TR Sogueros

Cañamiceros

USE Cañamenero

Capitanes

Carboneros

Carceleros

Carderos

Caritateros

Carniceros

UP Cortantes

UP Tajantes

UP Tajantes de barato

Carpinteros

TR Barqueros

Carreteros

Cedaceros

Cereros

Cerrajeros

Cesteros

Chapineros

TR Zapateros

Cirujanos

TR Barberos

TR Médicos
Clavarios

USE Clavazoneros

Clavazoneros

UP Clavarios

Cocheros

Cocineros

Cogedores (de hospital)

Colchoneros

Colmeneros

USE Abejeros

Comendadores

Comerciantes USE Mercaderes

Contadores (de la tabla de depósitos)

Coraceros

Cordoneros

Corredores de aceite UP Corredores de

ampolla

TR Corredores de caballos

TR Corredores de mulas

TR Corredores de oreja

TR Corredores de

percha

TR Corredores de redoma

TR Corredores de ropa

TR Mercaderes

TR Tenderos

Corredores de ampolla

USE Corredores de aceite

Corredores de bestias

USE Corredores de

caballos

USE Corredores de mulas

Corredores de caballos

UP Corredores de bestias

TR Corredores de aceite

TR Corredores de mulas

TR Corredores de oreja

TR Corredores de percha

TR Corredores de redoma

TR Corredores de ropa

TR Mercaderes

TR Tenderos

Corredores de mulas

UP Corredores de bestias

TR Corredores de aceite
TR Corredores de

caballos

TR Corredores de oreja

TR Corredores de percha

TR Corredores de redoma

TR Corredores de ropa

TR Mercaderes

TR Tenderos

Corredores de oreja

TR Corredores de aceite

TR Corredores de

caballos

TR Corredores de mulas

TR Corredores de percha

TR Corredores de redoma

TR Corredores de ropa

TR Mercaderes

TR Tenderos

Corredores de percha

TR Corredores de aceite

TR Corredores de

caballos

TR Corredores de mulas

TR Corredores de oreja

TR Corredores de redoma

TR Corredores de ropa

TR Mercaderes

TR Tenderos

Corredores de redoma

TR Corredores de aceite

TR Corredores de

caballos

TR Corredores de mulas

TR Corredores de oreja

TR Corredores de percha

TR Corredores de ropa

TR Mercaderes

TR Tenderos

Corredores de ropa

TR Corredores de aceite

TR Corredores de

caballos

TR Corredores de mulas

TR Corredores de oreja

TR Corredores de percha

TR Corredores de redoma

TR Mercaderes

TR Tenderos

Correos

TR Alquiladores de mulas

Scire. 8:1 (en.-jun. 2002) 131-145. 
TR Trajineros

Correos de a pie

Cortantes

USE Carniceros

Criados

Cronistas (del Reino de Aragón)

Cruceros

Cuberos

Cuchilleros

Curtidores

USE Zurradores

Deanes

Despenseros

Doctores en ambos derechos USE Doctores en derecho

Doctores en derecho UP Doctores en ambos derechos

TR Abogados fiscales TR Juristas

Doctores en medicina USE Médicos

Doradores

Drogueros

TR Boticarios

Encuadernadores

UP Atadores de libros TR Libreros

Empedradores

Enfermeros

Ensambladores

Entalladores

UP Entalladores de aljez

UP Entalladores de yeso y madera

Entalladores de aljez

USE Entalladores

Entalladores de yeso y madera

USE Entalladores

Escopeteros

TR Arcabuceros

TR Armeros

TR Ballesteros

TR Espaderos

TR Polvoristas

TR Puñaleros

Escribanos
Escribientes

Escritores

UP Escritores de libros

UP Escritores de libros de iglesia

Escritores de libros

USE Escritores

Escritores de libros de iglesia USE Escritores

Escuderos

Escudilleros

Escultores

Escultores de sellos

Esmaltadores de vidrio USE Vidrieros

Espaderos

UP Forjadores de espadas

TR Arcabuceros

TR Armeros

TR Ballesteros

TR Escopeteros

TR Polvoristas

TR Puñaleros

Esparteñeros

TR Cañameneros

TR Sogueros

Especieros

TR Herbolarios

Esportoneros

Estañeros

TR Buidadores

Estudiantes

Fabriqueros

Farmacéuticos

USE Boticarios

Físicos

USE Médicos

Forjadores de espadas

USE Espaderos

Freneros

Fusteros

Ganaderos

TR Cabreros

TR Pastores

TR Vaqueros

Gorreros

UP Maestros de hacer gorras
Guadamacileros

Guanteros

Guardas

Guarnicioneros

Herbolarios

TR Especieros

Herradores

USE Herreros

Herreros

UP Herradores

Hiladores de seda

TR Tejedores de sedas

Hombres de armas

Horneros

Imagineros

Impresores

UP Impresores de libros

Impresores de libros

USE Impresores

Infanzones

Ingenieros

Inquisidores

Jaboneros

Juristas

TR Abogados fiscales

TR Doctores en derecho

Justicias

Labradores

UP Agricultores

Lacayos

Lenceros

TR Pañeros

TR Traperos

Leñadores

Libreros

UP Mercaderes de libros

UP Mercaderes libreros

TR Encuadernadores

Limosneros

Lumineros

Maestres de capilla

Maestros

Maestros de cantería

USE Canteros

Maestros de canto

Maestros de enseñar a leer

USE Maestros de leer

Maestros de enseñar a escribir

Scire. 8: 1 (en.-jun. 2002) 131-145. 
y contar

USE Maestros de escribir y contar

Maestros de escribir y contar UP Maestros de enseñar a escribir y contar TR Maestros de leer

Maestros de esgrima

Maestros de gramática

Maestros de hacer barcas USE Barqueros

Maestros de hacer gorras USE Gorreros

Maestros de hacer pólvora USE Polvoristas

Maestros de hacer vidrio USE Vidrieros

Maestros de leer UP Maestros de enseñar a leer

TR Maestros de escribir y contar

Maestros de muchachos

Maestros de villa

USE Obreros de villa

Maestros en artes

Maestros en sagrada teología USE Maestros en teología

Maestros en santa teología USE Maestros en teología

Maestros en teología UP Maestros en sagrada teología

UP Maestros en santa teología

Maestros mayores (universidad)

Manteros

Mayorales

Mazoneros

UP Mazoneros de aljez

Mazoneros de aljez

USE Mazoneros

Médicos

UP Doctores en medicina

UP Físicos

TR Barberos

TR Cirujanos

Menestriles
Mercaderes

UP Comerciantes

TR Corredores de aceite

TR Corredores de

caballos

TR Corredores de mulas

TR Corredores de oreja

TR Corredores de percha

TR Corredores de redoma

TR Corredores de ropa

TR Tenderos

Mercaderes de libros

USE Libreros

Mercaderes de seda USE Sederos

Mercaderes de trapería USE Traperos

Mesoneros

TR Venteros

Moleros

Molineros

Músicos

UP Tañedores

TR Organistas

TR Violeros

Músicos de tañer vihuela UP Tañedores de vihuela

Naiperos

Notarios causídicos

Notarios públicos

UP Notarios públicos del número

Notarios públicos del número USE Notarios públicos

Notarios reales

Nuncios

Obispos

TR Arzobispos

Obreros de villa

UP Maestros de villa

TR Albañiles

Odreros

Olleros

Oracioneros

UP Rezadores de oraciones

Organistas

TR Músicos

TR Violeros
Pajes

Panaderos

Pañeros

UP Pañiceros

TR Lenceros

TR Traperos

Pañiceros

USE Pañeros

Papeleros

Parcheros

Pasamaneros

Pasteleros

Pastores

TR Cabreros

TR Ganaderos

TR Vaqueros

Pelaires

Pelliceros

UP Adobadores de cueros

TR Blanqueros

TR Pergamineros

TR Zurradores

Peloteros

Pergamineros

TR Blanqueros

TR Pelliceros

TR Zurradores

Pesadores

UP Pesadores del peso de harina

Pesadores del peso de harina USE Pesadores

Pescadores

Piedrapiqueros

Pintores

UP Artífices de pinturas

Plateros

Plegadores(de hospital)

UP Plegadores de ropa

Plegadores de ropa

USE Plegadores

Poceros

Polvoristas

UP Maestros de hacer

pólvora

TR Arcabuceros

TR Armeros

TR Ballesteros

TR Escopeteros

Scire. 8: 1 (en.-jun. 2002) 131-145. 
TR Espaderos

TR Puñaleros

Porteros

Presbíteros

TR Racioneros

Presbíteros racioneros

USE Racioneros

Priores

Puñaleros

TR Arcabuceros

TR Armeros

TR Ballesteros

TR Escopeteros

TR Espaderos

TR Polvoristas

Racioneros

UP Presbíteros racioneros

TR Presbíteros

Receptores del Santo Oficio

Rejoleros

Relojeros

Reposteros

Rezadores de oraciones

USE Oracioneros

Sacatierras

Sacerdotes

Sacristanes

Santeros

Sastres

TR Calceteros

Secretarios del Santo Oficio

Sederos

TR Mercaderes de seda

Serradores

Silleros

Sobreguardas

Sogueros

TR Cañameneros

TR Esparteñeros

Soldados

Sombrereros

TR Boneteros

Tablajeros

Tajantes

USE Carniceros

Tajantes de barato

USE Carniceros

Talegueros
Tañedores

USE Músicos

Tañedores de vihuela

USE Músicos de tañer vihuela

Tapiadores

Tapiceros

Tejeros

Tejedores de lanas

Tejedores de lienzos

Tejedores de linos

Tejedores de paños

Tejedores de sedas

TR Hiladores de seda

Tejedores de tafetanes

Tejedores de terciopelos

TR Velluteros

Tenderos

TR Comerciantes

TR Corredores de aceite

TR Corredores de

caballos

TR Corredores de mulas

TR Corredores de oreja

TR Corredores de percha

TR Corredores de redoma

TR Corredores de ropa

TR Mercaderes

Tesoreros

Tintureros

Tiradores de oro

Torcedores de seda

Torneros

TRabajadores

TRajinantes

USE Trajineros

TRajineros

UP Trajinantes

TR Alquiladores de mulas

TRaperos

UP Mercaderes de

trapería

TR Lenceros

TR Pañeros

TRasmudadores

TRatantes

Tundidores

Vaqueros

TR Cabreros
TR Ganaderos

TR Pastores

Veleros

Velluteros

TR Tejedores de terciopelos

Venteros

TR Mesoneros

Verdugaderos

Vergueros

Veterinarios

USE Albéitares

Vicarios

Vicecancilleres

Vidrieros

UP Esmaltadores de

vidrio

UP Maestros de hacer

vidrio

Vigoleros

Violeros

TR Músicos

TR Músicos de tañer

vihuela

TR Organistas

Zapateros

TR Chapineros

Zurradores

TR Blanqueros

TR Pelliceros

TR Pergamineros

Scire. 8: 1 (en.-jun. 2002) 131-145. 


\section{Bibliografía}

AENOR (1994). Documentación: normas fundamentales. Madrid: AENOR, 1994.

Álvarez Rubio, J. (1999). Profesiones y nobleza en la España del Antiguo Régimen. Madrid: Colegios Notariales de España, 1999.

Aranes Usandizaga, J. I. ; Landa Montenegro, C. (2000). Estrategias documentales para generar conocimiento. Representación y construcción de la realidad mediante un tesauro. // La gestión del conocimiento: retos y soluciones de los profesionales de la información: VIII Jornadas Españolas de Documentación. Bilbao, 2000. 633-638.

Blasco Martínez, R. M. y Maiso González, J. (1984). Las estructuras sociales en Zaragoza en el primer tercio del Siglo XVIII. Zaragoza: Institución Fernando el Católico, 1984.

Chaumier, J. (1986). Análisis y lenguajes documentales: El tratamiento lingüístico de la información documental. Barcelona: Mitre, 1986.

Currás, E. (1991). Thesauros: lenguajes terminológicos. Madrid: Paraninfo, 1991

García Marco, F. J. (1993). Las comunidades mudéjares en Calatayud en el Siglo XV. Calatayud: Centro de Estudios Bilbilitanos, 1993.

Gil Urdiciaín, B. (1996). Lenguajes documentales. // López Yepes, J. (coord.). Manual de Información y Documentación. Madrid: Pirámide, 1996. 325 -374.

Hernández Sandoica, E. (1995). Los caminos de la Historia: Cuestiones de historiografía y método. Madrid: Síntesis, 1995.

Martín Suquía, R. (coord.). (1993). Vocabulario de indización y consulta de la base de datos BADATOR. Bergara: IRARGI, Centro de Patrimonio Documental de Euskadi, 1993. Ikerlanak; núm. 5.

Oficina Internacional del Trabajo (1998). Tesauro OIT: Terminología del trabajo, el empleo y la formación. Ginebra: Oficina Internacional del Trabajo, 1998.

Pedraza Gracia, M. J. (1999). Lector, lecturas, bibliotecas... el inventario como fuente para su investigación histórica. // Anales de Documentación. 2 (1999)137-158

Rubio Liniers, M, C. (1999). Tesauro de Historia Contemporánea de España. Madrid: CINDOC, 1999.

San Vicente, A. (1998). Instrumentos para una historia social y económica del trabajo en Zaragoza en los siglos XV al XVIII. Zaragoza: Real Sociedad Económica Aragonesa de Amigos del País, 1988.

Slype G. Van (1991). Los lenguajes de indización: Concepción, construcción y utilización en los sistemas documentales. Madrid: Fundación Germán Sánchez Ruipérez, 1991

UNESCO (1984). Tesauro de la UNESCO: Lista estructurada de descriptores para la indización y la recuperación bibliográficas en las esferas de la educación, la ciencia, las ciencias sociales, la cultura y la comunicación. París: UNESCO, 1984.

ISOC. Tesauro ISOC de Economía. Madrid: CINDOC. URL:< http://pci204.cindoc.csic.es/tesauros/index.html/Tes_Econ.html >. Consultado: 2001-05-31.

Tesauro SPINES. Madrid: CINDOC. URL: <http://pci.cindoc.csic.es//tesauros/SpinTes/ Spines.htm >. Consultado: 2001-06-13. 